\title{
The CAESAR New Frontiers Comet Sample Return Mission
}

K. Nakamura-Messenger ${ }^{1}$, S. W. Squyres ${ }^{2}$, L. F. Pace ${ }^{1}$, S. Messenger ${ }^{1}$, D. F. Mitchell ${ }^{3}$, D. S. Lauretta ${ }^{4}$, D. P. Glavin ${ }^{3}$, M. Houghton ${ }^{3}$, A. G. Hayes ${ }^{2}$, T. Nakamura ${ }^{5}$, J. P. Dworkin ${ }^{3}$, A. Nguyen ${ }^{6}$, S. Clemett ${ }^{6}$, Y. Furukawa $^{5}$, Y. Kimura ${ }^{7}$, A. Takigawa ${ }^{8}$, G. Blake ${ }^{9}$, T. J. Zega ${ }^{4}$, M. Mumma ${ }^{3}$, S. Milam ${ }^{3}$, C. D. K. Herd ${ }^{10}$, J. Mitchell ${ }^{1}$, and the CAESAR Project Team.

1. ARES/EISD NASA Johnson Space Center, Houston, TX, USA.

2. Cornell University, Ithaca, NY, USA.

3. NASA Goddard Space Flight Center, Greenbelt, MD, USA.

4. University of Arizona, Tucson, AZ, USA.

5. Tohoku University, Sendai, Japan.

6. JETS, NASA Johnson Space Center, Houston, TX.

7. Hokkaido University, Sapporo, Japan.

8. Kyoto University, Kyoto, Japan.

9. California Institute of Technology, Pasadena, CA, USA.

10. University of Alberta, Edmonton, AB, Canada, Japan.

The Comet Astrobiology Exploration Sample Return (CAESAR) mission is one of two finalists selected by NASA for Phase A study in the New Frontiers program. CAESAR will acquire a minimum of $80 \mathrm{~g}$ of material from the surface of comet 67P/Churuyumov-Gerasimenko and return it to Earth for laboratory analysis. CAESAR preserves much of the science of a cryogenic sample return by retaining volatiles in a dedicated reservoir securely separated from the solid sample. Comet 67P was selected based on its favorable orbital geometry and the risk reduction and scientific context provided by ESA's Rosetta mission.

CAESAR's objectives are to understand the origins of the Solar System starting materials and how these components came together to form planets and give rise to life. We also seek to resolve the conflicting views of comet origins arising from the Stardust and Rosetta missions. While the $>1 \mu \mathrm{m}$ solids returned by Stardust originated in the hot, inner solar nebula, measurements by Rosetta suggest $67 \mathrm{P}$ volatiles formed at cryogenic temperatures and remained unchanged for billions of years. This dichotomy provides the rationale for returning both solid and gaseous samples.

Analyses of returned samples will determine the nature and abundances of interstellar materials and the histories and ages of refractory solar nebula condensates. They will trace the evolution of volatile reservoirs, delineate chemical pathways that led from simple interstellar species to complex and prebiotic molecules, and constrain the geological and dynamic evolution of 67P. And they will evaluate the potential role of comets in delivering water and organics to the early Earth. These goals will be achieved by sample analyses that link macroscopic properties of the comet with microscale mineralogy, chemistry, and isotopic studies of volatiles and solids. These analyses can be performed in terrestrial laboratories with orders of magnitude greater sensitivity and precision than possible with spacecraft instrumentation.

The CAESAR spacecraft, provided by Orbital ATK, uses NEXT-C thrusters for solar electric propulsion. Outbound cruise includes an Earth flyby, arriving at 67P in December 2028. Navigation, sample site selection, and sample documentation are enabled by the CAESAR camera suite, provided by Malin Space Science Systems. Remote imaging will give geological context for the sample and document changes to 67P after two perihelion passages. 
The Sample Acquisition System (SAS), developed by Honeybee Robotics, collects comet nucleus material during a 5 second touch-and-go (TAG) event. Upon surface contact, high-purity $\mathrm{N}_{2}$ gas directs particles into a $1.5 \mathrm{~L}$ Au-plated sample container. SAS performance tests at zero gravity and in vacuum at the NASA Glenn Zero Gravity Research Facility routinely collected over $300 \mathrm{~g}$ of comet simulant. Sample collection is verified by imaging the sample container interior and sample mass measurement using a load cell. Following sample verification, the SAS is inserted into the sample containment system (SCS). The SCS is then hermetically sealed with a knife-edge seal and copper gasket.

After sealing the SCS, the sample is warmed to near-perihelion temperatures, allowing volatiles to sublime. The sublimated volatiles are passively cryopumped into a separate Au-coated gas containment system (GCS) radiator-cooled to below $-60^{\circ} \mathrm{C}$. After determining that the volatile transfer is complete by means of pressure and $\mathrm{H}_{2} \mathrm{O}$ partial pressure sensors, the GCS-SCS valve is closed and the SCS is vented to space until just before Earth return.

The CAESAR SRC is provided by JAXA and its design is based upon the SRC flown on the Hayabusa and Hayabusa2 spacecraft. As with these missions, CAESAR's SRC jettisons its heat shield while still in flight on the parachute, minimizing heat soak back. The sample is insulated with phasechange material to maintain the sample at below $0{ }^{\circ} \mathrm{C}$ until it is recovered at the Utah Test and Training Range on November 20, 2038.

To achieve the CAESAR mission goals, the condition of the sample must be preserved and documented through collection, recovery, and curation within the resources of the NASA New Frontiers 4 program. We have taken our experiences from OSIRIS-REx, Hayabusa, Genesis, Stardust, and Apollo, and applied them to the unique sample returned from a comet surface. By necessity, CAESAR's protocols to prevent sample alteration go well beyond previous sample return missions. Comet solids will most likely consist of an assemblage of highly unequilibrated components from the early solar nebula, and must be kept cold and dry to avoid reactions between grains and with any adventitious moisture. Amorphous silicates in cometary interplanetary dust particles (IDPs) alter to hydrated silicates in hours in room temperature water. Even brief exposure of the sample to liquid water could confound attempts to determine if aqueous activity occurred in 67P. CAESAR's sample recovery, storage, handling procedures and facilities will be designed to protect the samples from contamination, temperature excursions, and moisture that could induce alteration in the sample. The facilities will enable the careful documentation and processing of the sample using methods suitable for microanalytical studies of small particles, volatiles, and organics.

We based CAESAR's science investigation on existing capabilities and instrumentation. We can achieve our sample analysis goals using current technologies for coordinated sample analyses that link macroscopic properties of the comet with microscale mineralogy, chemistry, and isotopic studies of volatiles and solids. However, since CAESAR samples will be returned 20 years from now, future researchers will address these fundamental science goals using ever more capable instruments and new scientific insights, providing an invaluable scientific resource for the worldwide planetary science community for generations to come. 